OPEN ACCESS

Edited by:

Matthias Lauth,

Philipps University of Marburg,

Germany

Reviewed by:

Tamara Caspary,

Emory University, United States

Zizhang Zhou,

Shandong Agricultural University,

China

*Correspondence:

Jianhang Jia

Jianhang.jia@uky.edu

Jin Jiang

jin.jiang@UTSouthwestern.edu

Specialty section:

This article was submitted to

Signaling,

a section of the journal

Frontiers in Cell and Developmental

Biology

Received: 03 January 2022

Accepted: 23 February 2022

Published: 07 March 2022

Citation:

Jia $J$ and Jiang J (2022) Regulation of Smoothened Trafficking and

Abundance in Hedgehog Signaling.

Front. Cell Dev. Biol. 10:847844.

doi: 10.3389/fcell.2022.847844

\section{Regulation of Smoothened Trafficking and Abundance in Hedgehog Signaling}

\author{
Jianhang Jia ${ }^{1 *}$ and Jin Jiang ${ }^{2 *}$ \\ ${ }^{1}$ Department of Molecular and Cellular Biochemistry, Markey Cancer Center, University of Kentucky College of Medicine, \\ Lexington, KY, United States, ${ }^{2}$ Department of Molecular Biology, UT Southwestern Medical Center, Dallas, TX, United States
}

The GPCR-family protein Smoothened (Smo) is essential for Hedgehog $(\mathrm{Hh})$ signal transduction in both insects and vertebrates. The regulation of subcellular localization and abundance of Smo is a critical step in Hh signaling. Recent studies have demonstrated that $\mathrm{Smo}$ is subjected to ubiquitination mediated by multiple E3 ubiquitin ligases, leading to Smo endocytosis and subsequent degradation through the proteasome- and lysosomemediated pathways in Drosophila. Ubiquitination of Smo also promotes its ciliary exit in mammalian cells. Hh inhibits Smo ubiquitination by blocking E3 ligase recruitment and promoting Smo deubiquitination through the ubiquitin-specific protease 8 (USP8) in Drosophila. Inhibition of Smo ubiquitination by $\mathrm{Hh}$ promotes Smo cell surface accumulation in Drosophila and ciliary accumulation in mammalian cells. Interestingly, Hh also induces sumoylation of Smo in both Drosophila and mammalian cells, which promotes Smo cell surface/ciliary accumulation. This review focuses on how ubiquitination and sumoylation regulate Smo intracellular trafficking and abundance and how these processes are regulated by $\mathrm{Hh}$.

Keywords: hedgehog, smoothened, GPCR, ubiquitination, sumoylation, endocytosis, primary cilium, Smurf

\section{INTRODUCTION}

The Hedgehog $(\mathrm{Hh})$ morphogen controls embryonic development and adult tissue homeostasis in species ranging from insects to mammals (Ingham and McMahon, 2001; Ingham et al., 2011; Jiang and Hui, 2008). Malfunction of Hh signaling has been implicated in many human disorders, including birth defect and cancer (Jiang, 2021; Pasca di Magliano and Hebrok, 2003; Taipale and Beachy, 2001; Villavicencio et al., 2000). Hh signal transduction is largely conserved among species (Figure 1), especially at the plasma membrane, which involves the Hh receptor Patched (Ptc) and coreceptor interference of Hh (Ihog) (Camp et al., 2010; Zheng et al., 2010), and the signal transducer Smoothened (Smo). Smo, a G protein-coupled receptor (GPCR) of the Frizzled-class (class-F), is essential for transducing the Hh signal across the plasma memberane in both insects and vertebrates (Ingham and McMahon, 2001; Jia and Jiang, 2006; Zhao et al., 2007; Jiang and Hui, 2008). Abnormal Smo activation results in basal cell carcinoma (BCC), medulloblastoma, and other types of cancer (Jiang, 2021), making it an attractive therapeutic target.

In Drosophila, the Hh signal is transduced at the plasma membrane, where the receptor complex Ptc-Ihog and Smo are located. Hh binding to Ptc-Ihog relieves Smo inhibition by Ptc, resulting in Smo cell surface accumulation and activation, and subsequent activation of the Gli-family of zinc finger transcription factor Cubitus interruptus (Ci) that regulates the expression of $\mathrm{Hh}$ target genes. In mammals, Hh signal is transduced at primary cilia where Smo and Gli proteins are accumulated 


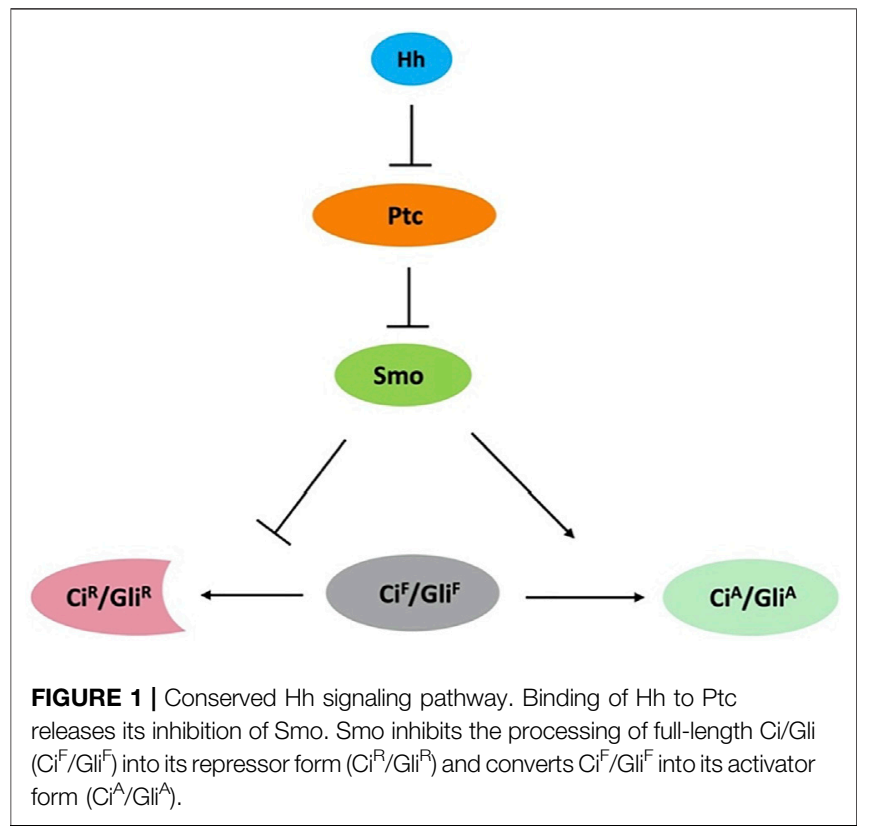

during pathway activation (Bangs and Anderson, 2017). Smo is subjected to post-translational modifications (PTMs). Previous studies have shown that Hh induces phosphorylation of Smo by protein kinase A (PKA), casein kinase 1 (CK1), casein kinase 2 (CK2), and G protein-coupled receptor kinase 2 (GRK2) as well as atypical PKC (aPKC) in Drosophila (Chen and Jiang, 2013; Jiang et al., 2014; Li et al., 2014; Jiang and Jia, 2015; Li et al., 2016). Mammalian Smo ciliary localization and activation are also regulated through phosphorylation mediated by CK1 and GRK2 (Chen et al., 2011; Arveseth et al., 2021). Differential phosphorylation and conformational change in Smo mediate the transduction of $\mathrm{Hh}$ activity gradient (Jia et al., 2004; Zhao et al., 2007; Chen et al., 2011; Fan et al., 2012). Recent studies have demonstrated that the cell surface accumulation of Smo is regulated by ubiquitination-mediated endocytosis, which is opposed by Smo phosphorylation as well as Smo sumoylation (Zhang and Jiang, 2021). This review focuses on Smo ubiquitination and sumoylation that are the main mechanisms underlying the regulation of Smo trafficking and abundance. We also discuss the similarities and differences in the regulation of Smo between Drosophila and mammals where $\mathrm{Hh}$ signaling occur in the primary cilium (Bangs and Anderson, 2017).

\section{REGULATION OF DROSOPHILA SMO TRAFFICKING AND ABUNDANCE BY MULTIPLE E3 UBIQUITIN LIGASES}

Ubiquitination is one of most prevalent PTMs that dictates the fate and function of many cellular proteins (Hershko and Ciechanover, 1998; Popovic et al., 2014). Attachment of ubiquitin to a protein substrate is catalyzed sequentially by ubiquitin activating enzyme (E1), ubiquitin conjugating enzyme (E2), and ubiquitin-protein ligase (E3) that recognizes the protein substrate (Hershko and Ciechanover,
1998). Studies on GPCRs have demonstrated that activation of receptors often stimulates receptor endocytosis mediated by ubiquitination, which plays a role in intracellular signaling in addition to pathway desensitization (Shenoy et al., 2001; Wojcikiewicz, 2004; Irannejad and von Zastrow, 2014). However, unlike other GPCRs whose endocytosis and subsequent degradation is induced upon ligand binding, Smo internalization and cell surface clearance are inhibited in response to Hh stimulation (Denef et al., 2000).

Initial genetic experiments found that inactivation of the Ubiquitin-Activating Enzyme, Uba1, as well as multiple endocytic pathway components in Drosophila wing imaginal discs resulted in ectopic cell surface accumulation of Smo in anterior compartment cells that are not exposed to Hh, implying that ubiquitination-mediated endocytosis is involved in removing Smo from the cell surface (Li et al., 2012; Xia et al., 2012). Further biochemical studies revealed that Smo is subjected to both multiubiquitination and poly-ubiquitination and is degraded by both proteasome- and lysosome-dependent mechanisms, and that Smo ubiquitination is inhibited by $\mathrm{Hh}$ through PKA/CK1mediated phosphorylation of Smo C-terminal intracellular tail (SmoCT) (Li et al., 2012; Xia et al., 2012).

Much effort has since been devoted to the identification of Smo E3 ligases. The complexity comes from the fact that multiple E3 ligases appear to be involved and therefore, not a single Smo E3 ligase has been identified through the conventional genetic screen. Using a cell-based Smo ubiquitination assay that is sensitive to perturbation, a recent study identified three HECT-domain containing E3 ligases of the Smurf family (also called the Nedd 4 family), including Smurf, Nedd4, and Su(dx), whose RNAi reduced Smo ubiquitination in Schneider 2 (S2) cells (Li et al., 2018b). Both loss- and gain-of-function studies in Drosophila wing discs suggested that Smurf plays major role while Nedd4 and $\mathrm{Su}(\mathrm{dx})$ play minor role in the regulation of Smo cell surface accumulation (Li et al., 2018b). In the absence of Hh, these E3s bind SmoCT through their HECT domains to promote Smo ubiquitination, internalization, and degradation whereas Hh-induced phosphorylation of SmoCT by PKA/CK1 inhibits E3 recruitment and thus Smo ubiquitination, leading to Smo cell surface accumulation (Figure 2A) (Li et al., 2018b).

Interestingly, the binding of Smurf to Smo is promoted by GRK2, which phosphorylates the N-terminal region of Smurf to release an intramolecular autoinhibitory interaction between the $\mathrm{N}$-terminal region and the C-terminally located HECT domain (Li et al., 2018b). This regulatory mechanism could explain why Smo was stabilized in GRK2 mutant cells (Chen et al., 2010). Release of the Smurf family of E3s from Smo increases their accessibility to Ptc and Hh further stimulates the binding of these E3s to Ptc to promote its ubiquitination and endocytosis ( $\mathrm{Li}$ et al., $2018 b$ ), which accounts for the increased internalization of Ptc in response to $\mathrm{Hh}$ (Denef et al., 2000).

A genetic modifier screen identified another HECT-domain and RCC1-like domains (RLDs) containing E3 ligase HERC4 as regulator of Smo ubiquitination and cell surface abundance (Jiang et al., 2019). Like the Smurf family E3s, HERC4 interacted with SmoCT through its HECT domain as well as RCC1-like domains, which is inhibited by Hh-induced phosphorylation of SmoCT by 
A

A $\quad-H h$

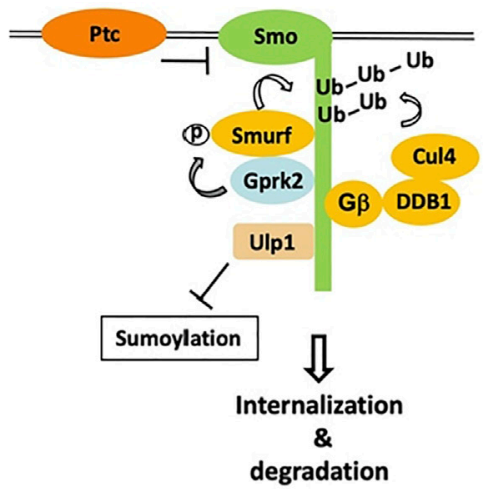

B

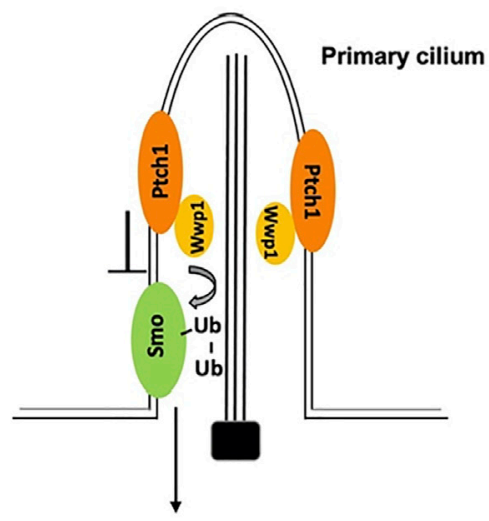

$+\mathrm{Hh}$
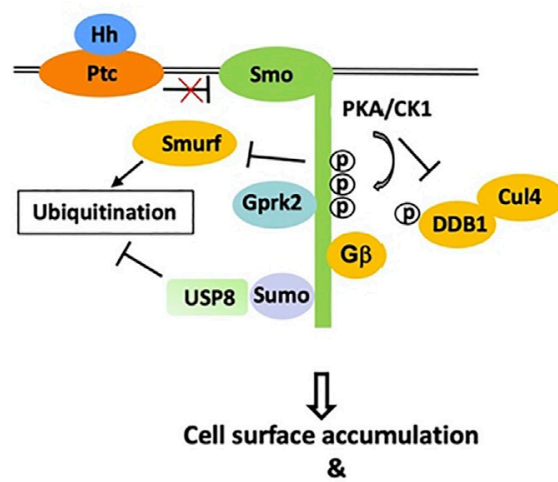

signal transduction

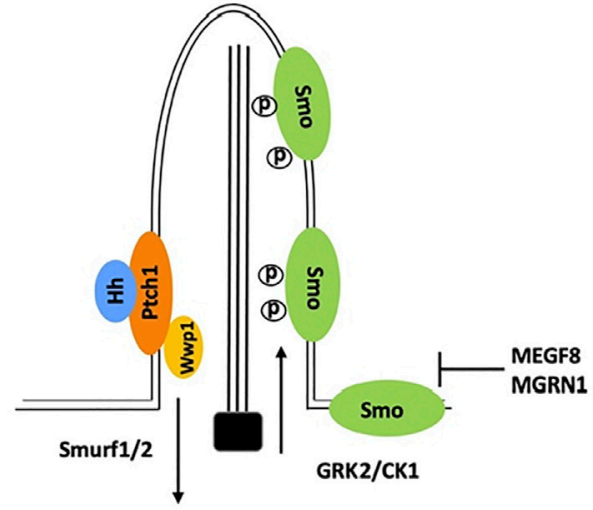

FIGURE 2 | Ubiquitination and sumoylation are involved in the regulation of Smo trafficking and stability. (A) In Drosophila cells not exposed to Hh, Smo is ubiquitinated by the Smurf family of E3s and other E3s including Cul4-DDB1-G 3 E3 complex, which promotes Smo endocytosis and degradation. Phosphorylation by Gprk2 stimulates the binding of Smurf to Smo. Binding of Ulp1 to Smo blocks its sumoylation. Hh stimulates phosphorylation of Smo and DDB1 by PKA to dissociate Smurf and Cul4-DDB1, respectively, and induces Smo sumoylation to recruit USP8, which blocks Smo ubiquitination, leading to Smo cell surface accumulation. (B) In mammalian cells, Wwp1, which is localized in primary cilia through its binding to Patch1, promotes Smo ubiquitination and ciliary exit. Hh inhibits Smo ubiquitination by promoting ciliary exit of Ptch1 and Wwp1, leading to ciliary accumulation of Smo. A membrane-associated E3 complex MEGF8/MGRN1 promotes the degradation of Smo to modulate cells' sensitivity to $\mathrm{Hh}$.

PKA/CK1. Another study showed that mammalian HERC4 is downregulated and negatively correlated with Smo in non-small cell lung cancer (NSCLC) patient samples and that knockdown of HERC4 activated Hh pathway and promoted NSCLC cell proliferation (Sun et al., 2019).

Finally, an in vivo RNAi screen for genes whose loss of function resulted in Smo accumulation identified Cullin4 (Cul4) and its binding partner DDB1 as negative regulators of Smo accumulation in wing discs (Li et al., 2018a). Cul4 forms multi-subunit E3 ubiquitin ligase complexes in which DDB1 bridges Cul4 to multiple DDB1-binding WD40 (DWD) proteins that recognize specific substrates (Angers et al., 2006). Biochemical studies revealed that Smo recruits Cul4-DDB1 through multiple $\beta$ subunits of trimeric $G$ proteins $(G \beta s)$ to promote its ubiquitination and turnover, and that Hh signaling recruited the catalytic subunit of PKA (PKAc) to Smo to phosphorylate DDB1, which dissociates Cul4-DDB1 from G $\beta$ and thus inhibits Cul4-mediated Smo ubiquitination (Figure 2A) (Li et al., 2018a).

\section{REGULATION OF MAMMALIAN SMO CILIARY TRAFFICKING AND ABUNDANCE BY UBIQUITINATION}

In mammal cells, Hh induces reciprocal trafficking of Ptc and Smo with Ptc moving out and Smo accumulating in primary cilia (Corbit et al., 2005; Rohatgi et al., 2007). Recent studies found that 
Smo ubiquitination promoted its ciliary exit through IFT27 and BBSome components (Desai et al., 2020; Shinde et al., 2020). Blocking of Smo ubiquitination by pharmacologically inhibiting E1 activity or by mutating two ubiquitin attachment sites in Smo resulted in Smo ciliary accumulation in the absence of $\mathrm{Hh}$ whereas fusing a ubiquitin moiety to Smo inhibited its ciliary accumulation in the presence of Hh (Desai et al., 2020). Using CRISPR screen of candidate genes, a subsequent study by the same group identified the HECT domain E3 ligase Wwp1 as essential for Smo ubiquitination and ciliary exit (Lv et al., 2021). Interestingly, Wwp1 is localized in primary cilia through its interaction with Ptc and Ptc-mediated ciliary localization of Wwp1 is critical for promoting Smo ubiquitination and ciliary exit (Lv et al., 2021). Accordingly, Hh-induced ciliary exit of Ptc removes Wwp1 from the primary cilia, which may prevent Smo ubiquitination, leading to Smo ciliary accumulation (Figure 2B). Wwp1 is related to the Drosophila Smurf family of E3s and its closest homolog in Drosophila is $\mathrm{Su}(\mathrm{dx})$, followed by Nedd 4 and Smurf. Hence, ubiquitination (Reyes-Turcu et al., 2009) mediated Smo trafficking in Drosophila and mammals is controlled by closely related E3 ubiquitin ligases. It will be interesting to determine whether ubiquitination regulates Smo ciliary exit directly by modulating its binding to BBSome and/or other IFT proteins or indirectly by promoting its endocytosis near the ciliary base, a mechanism proposed for ciliary removal of Ptc by Smurf1/2 (Figure 2B) (Yue et al., 2014).

Smo ubiquitination not only regulates its ciliary trafficking but also modulates its overall abundance. Aside from the demonstrated role of HERC4 in the regulation of mammalian Smo ubiquitination and stability in cultured NIH3T3 cells and NSCLC cells (Jiang et al., 2019; Sun et al., 2019), a genome-wide screen for modifiers of $\mathrm{Hh}$ pathway activity in cultured cells identified a pair of genes encoding a transmembrane protein MEGF8 and a RING family E3 ligase MGRN1 whose loss-offunction resulted in an increased response to Shh due to elevated Smo levels at the cell surface and primary cilia (Kong et al., 2020). MGRN1and MEGF8 formed a membrane localized E3 ligase complex to catalyze the ubiquitination of Smo, leading to its degradation. Mice homozygous for Megf8 or Mgrn1 mutation exhibited increased Smo abundance and elevated sensitivity to Hh signaling whereas mice heterozygous for either Megf8 or Mgrn1 mutation were normal but double heterozygous embryos exhibited an incompletely penetrant congenital heart defects (CHDs), revealing delicate genetic interactions between Megf8 and Mgrn1 that affect Hh signaling strength through modulating Smo abundance (Kong et al., 2020).

\section{REGULATION OF SMO UBIQUITINATION BY DUBS}

Ubiquitination is a dynamic process that is reversed by deubiquitinating enzymes (Dubs) (Reyes-Turcu et al., 2009; Komander and Rape, 2012). Several Dubs have been implicated in the regulation of Smo ubiquitination. Loss-offunction of USP8 (also called UBPY) in Drosophila wing discs attenuated Smo accumulation in cells exposed to $\mathrm{Hh}$ whereas overexpression of USP8 led to ectopic accumulation of Smo and Hh pathway activation (Li et al., 2012; Xia et al., 2012). Consistent with these in vivo observations, overexpression of USP8 in S2 cells diminished Smo ubiquitination whereas knockdown of USP8 attenuated Hh-induced reduction of Smo ubiquitination (Li et al., 2012; Xia et al., 2012). Interestingly, the interaction between USP8 and Smo is enhanced by $\mathrm{Hh}$ but not by PKA/CK1mediated phosphorylation of SmoCT, suggesting that $\mathrm{Hh}$ regulates USP8 through a PKA/CK1-independent mechanism (Li et al., 2012; Xia et al., 2012).

A genetic modifier screen for genes that modulate the wing phenotype caused by expression of a dominant negative Smo $\left(\mathrm{Smo}^{\mathrm{DN}}\right.$ ) identified UCHL5 (thiol protease class of DUB whose mammalian counterpart is $\mathrm{UCH} 37$ ) as a regulator of Smo ubiquitination and cell surface expression (Zhou et al., 2018; Jiang et al., 2019). UCHL5 binds Smo and promotes its deubiquitination and cell surface accumulation, and the interaction between UCHL5 and Smo is enhanced by $\mathrm{Hh}$ (Zhou et al., 2018). Knockdown of UCH37 by RNAi reduced Smo level and Hh pathway activity in NIH3T3 cells, raising the possibility that UCHL5/UCH37 may play a conserved role in modulating Smo ubiquitination and turnover (Zhou et al., 2018).

\section{REGULATION OF SMO TRAFFICKING AND ABUNDANCE BY SUMOYLATION}

Similar to protein ubiquitination, sumoylation is another reversible covalent modification that controls many cellular processes involved in protein trafficking and stability control (Geiss-Friedlander and Melchior, 2007). During the sumoylation process, small ubiquitin-related modifier (SUMO) is attached to a target protein, which requires the activation by a SUMOactivation enzyme (E1) and subsequent SUMO transfer by a SUMO-conjugating enzyme (E2). A specific SUMO ligase (E3) is responsible for recognizing the target protein (Johnson, 2004). SUMO molecules can be removed by SUMO-specific isopeptidases (desumoylation enzymes).

Recent studies have linked the SUMO pathway to the regulation of Smo trafficking and abundance (Figure 2A) (Ma et al., 2016; Zhang et al., 2017). In vivo RNAi screens in Drosophila identified several components of the SUMO pathway whose knockdown modulated the wing phenotypes caused by expressing Smo ${ }^{\mathrm{DN}}$ in wing discs. Whereas RNAi of Ubc9, a SUMO-conjugating enzyme E2, PIAS, a SUMO-protein ligase E3, and Smt3, the SUMO isoform in Drosophila, enhanced $\mathrm{Smo}^{\mathrm{DN}}$-induced defects, inactivation of Ubiquitin-like protease 1 (Ulp1) partially suppressed the phenotypes (Ma et al., 2016; Zhang et al., 2017). Consistently, inactivation of Ubc9, PIAS, or Smt3 reduced Hh-induced Smo accumulation and pathway activity in both wing discs and cell cultures (Ma et al., 2016; Zhang et al., 2017). In addition, overexpression of Ulp1 decreased whereas knockdown of Ulp1 increased Smo levels (Ma et al., 2016; Zhang et al., 2017). Hh stimulated sumoylation of Smo mainly on Lys 851, which conforms the sumoylation consensus site (Ma et al., 2016). Substitution of Lys 851 to $\operatorname{Arg}\left(\mathrm{Smo}^{\mathrm{K} 851 \mathrm{R}}\right.$ ) greatly diminished Hh-induced Smo sumoylation, reduced Smo 
stability in cultured cells, and attenuated Hh-induced Smo accumulation and pathway gene expression in wing discs $(\mathrm{Ma}$ et al., 2016). The Hh signaling defect caused by the K851R mutation was fully rescued by fusing a SUMO moiety to the C-terminal end of the Smo variant (Ma et al., 2016), demonstrating that sumoylation of Smo at Lys 851 promotes Smo accumulation and Hh pathway activity.

Although binding of UBC9 and PIAS to Smo was undetectable regardless of whether $\mathrm{Hh}$ was present or not, Ulp1 interacted with SmoCT in a manner inhibited by Hh signaling, suggesting that Hh stimulates Smo sumoylation mainly by inhibiting the recruitment of the desumoylation enzyme (Ma et al., 2016; Zhang et al., 2017). Like the regulation of USP8-Smo interaction, Ulp1-Smo association and consequently, Smo sumoylation were not regulated by PKA-CK1 mediated phosphorylation of SmoCT (Ma et al., 2016; Zhang et al., 2017), suggesting a link between Smo sumoylation and USP8mediated Smo deubiquitination. Indeed, Smo sumoylation recruited USP8/UBPY through the Sumo-interacting domain (SIM) present in USP8/UBPY to antagonize Smo ubiquitination, leading to increased cell surface accumulation of Smo (Ma et al., 2016). Interestingly, Kurtz (Krz), the Drosophila $\beta$-arrestin 2 homolog, blocks Smo sumoylation and prevents its cell surface accumulation (Zhang et al., 2017). Inactivation of Krz decreased the interaction between Smo and Ulp1, suggesting that Krz regulates the sumoylation of Smo through facilitating Smo-Ulp1 interaction (Zhang et al., 2017).

Sumoylation is also involved in the regulation of mammalian Smo trafficking and Shh pathway activity (Ma et al., 2016). In NIH3T3 cells, SUMO-conjugation to Smo was stimulated by Shh but inhibited by overexpression of a mammalian desumoylation enzyme SENP1. Consistent with the notion that sumoylation regulates Shh pathway activity at the level of Smo, overexpression of SENP1 inhibited Shh pathway activity in wild type but not in Sufu mutant MEF cells. Overexpression of SENP1 in NIH3T3 cells inhibited Shh-induced Smo ciliary accumulation and Shh pathway activity, which was reversed by fusion of SUMO to the C-terminal end of Smo (Smo-SUMO). Furthermore, Smo-SUMO exhibited constitutive ciliary accumulation and Shh pathway activity, suggesting that sumoylation of Smo promotes its ciliary localization and activation. However, the sumoylation site(s) on mammalian Smo remains unidentified. In addition, whether sumoylation of Smo promotes its ciliary localization by antagonizing its ubiquitination awaits to be determined. In both Drosophila and mammals, how Hh stimulates Smo sumoylation remains a mystery.

\section{REGULATION OF SMO BY ENDOCYTIC PATHWAY COMPONENTS}

In support of the notion that ubiquitin-mediated endocytic trafficking regulates Smo, several components in the endocytic pathway have been shown to regulate Smo abundance. Inactivation of HGF-regulated tyrosine kinase substrate (Hrs), Tsg101, Avalanche (Avl), Rab5, endosomal sorting complex required for transport complex-II and III (ESCRT-II and ESCRT-III) in Drosophila wing discs resulted in accumulation of Smo in the absence of Hh (Li et al., 2012; Fan et al., 2013; Yang et al., 2013; Jiang et al., 2018). Hrs interacts with Smo and promotes its ubiquitination (Fan et al., 2013). In addition, the ESCRT-III core subunits, VPS32 (also known as Shrub in Drosophila, SNF7 in yeast, and CHMP4 in mammal) and VPS20 (CHMP6 in mammals) regulate Smo stability intracellularly (Jiang et al., 2018). Surprisingly, Smo is accumulated in phosphorylated and active forms in cells defective in ESCRT-III function, which does not rely on $\mathrm{Hh}$ stimulation, indicating that Smo can exhibit Hh-independent activity when accumulated at high levels in a specific intracellular compartment. Furthermore, a Krz-mediated pathway, operating in parallel to endocytosis, directs Smo to the ESCRT-III/ multivesicular body (MVB), leading to the high accumulation and activation of Smo when ESCRT-III function is inactivated (Jiang et al., 2018). Of note, the ESCRT machinery has also been shown to regulate the secretion and long-range Hh signaling (Matusek et al., 2014), suggesting that the ESCRT machinery regulates Hh signaling in both signal sending and receiving cells.

\section{CONCLUSION AND PERSPECTIVES}

Many scaffolding proteins and enzymes are involved in Smo ubiquitination, sumoylation, and intracellular trafficking, as discussed in this review; however, how these events are sophisticatedly controlled by $\mathrm{Hh}$ signaling has not been fully understood. Because vertebrate Hh signaling occurs in primary cilia and may utilize different mechanisms to regulate Smo, further studies in vertebrate systems are needed to explore the link between the various enzymes involved in ubiquitination and sumoylation and their possible involvement in human diseases. For example, while phosphorylation of Drosophila Smo (dSmo) by PKA inhibits its ubiquitination, mammalian Smo (mSmo) is not phosphorylated by PKA and whether phosphorylation of $\mathrm{mSmo}$ by other kinases such as CK1 and GRK2 regulates its ubiquitination remains to be determined. How $\mathrm{mSmo}$ sumoylation is stimulated by $\mathrm{Hh}$ and whether mSmo sumoylation also inhibits its ubiquitination remain to be explored. Although targeted expression of a UbK63-specific Dub could promote mSmo ciliary accumulation (Shinde et al., 2020; Lv et al., 2021), whether Shh stimulates binding of a $\operatorname{Dub}(\mathrm{s})$ to antagonize $\mathrm{mSmo}$ ubiquitination remains to be determined. It is interesting to note that $\mathrm{Hh}$ stimulates the binding of PKA catalytic subunit (PKAc) to SmoCT in both Drosophila and mammalian systems (Li et al., 2014; Ranieri et al., 2014; Arveseth et al., 2021). While binding of PKAc to $\mathrm{dSmo}$ is thought to promote $\mathrm{dSmo}$ phosphorylation and sequester PKAc away $\mathrm{Ci}$, binding of PKAc to a PKI motif in vertebrate SmoCT has been shown to sequester PKAc away from Gli2 and Gli3 and thus inhibit Gli phosphorylation and processing (Happ et al., 2021). It would be interesting to determine whether a similar PKI motif is present in $\mathrm{dSmo}$ and contributes its inhibition of $\mathrm{Ci}$ phosphorylation.

The mechanisms underlying Smo regulation are the subject of intense interest because aberrant Smo activation contributes to 
many type of human cancer including basal cell carcinoma and medulloblastoma (Xie et al., 1998; Yang et al., 2010; Jiang, 2021), making Smo an attractive therapeutic target as exemplified by the U.S. FDA approved drugs, such as vismodegib, sonidegib, and glasdegib for the treatment of cancers known to be driven by Smo activation (Guha, 2012; Sekulic and Von Hoff, 2016; Pietrobono and Stecca, 2018; Bohl et al., 2019). The regulation of Smo intracellular trafficking and abundance is a major mechanism controlling Hh pathway activity. Future studies of additional players involved in the regulation of Smo ubiquitination, sumoylation, and trafficking, as well as elucidating their functional and regulatory relationships, may provide new avenues for developing novel cancer therapeutics.

\section{REFERENCES}

Angers, S., Li, T., Yi, X., MacCoss, M. J., Moon, R. T., and Zheng, N. (2006). Molecular Architecture and Assembly of the DDB1-Cul4a Ubiquitin Ligase Machinery. Nature 443, 590-593. doi:10.1038/nature05175

Arveseth, C. D., Happ, J. T., Hedeen, D. S., Zhu, J.-F., Capener, J. L., Klatt Shaw, D., et al. (2021). Smoothened Transduces Hedgehog Signals via Activity-dependent Sequestration of PKA Catalytic Subunits. Plos Biol. 19, e3001191. doi:10.1371/ journal.pbio.3001191

Bangs, F., and Anderson, K. V. (2017). Primary Cilia and Mammalian Hedgehog Signaling. Cold Spring Harb Perspect. Biol. 9, a028175. doi:10.1101/cshperspect. a028175

Bohl, S. R., Bullinger, L., and Rücker, F. G. (2019). New Targeted Agents in Acute Myeloid Leukemia: New Hope on the Rise. Ijms 20, 1983. doi:10.3390/ ijms20081983

Camp, D., Currie, K., Labbé, A., van Meyel, D. J., and Charron, F. (2010). Ihog and Boi Are Essential for Hedgehog Signaling in Drosophila. Neural Dev. 5, 28. doi:10.1186/1749-8104-5-28

Chen, Y., and Jiang, J. (2013). Decoding the Phosphorylation Code in Hedgehog Signal Transduction. Cell Res 23, 186-200. doi:10.1038/cr.2013.10

Chen, Y., Li, S., Tong, C., Zhao, Y., Wang, B., Liu, Y., et al. (2010). G ProteinCoupled Receptor Kinase 2 Promotes High-Level Hedgehog Signaling by Regulating the Active State of Smo through Kinase-dependent and Kinaseindependent Mechanisms in Drosophila. Genes Dev. 24, 2054-2067. doi:10. 1101/gad.1948710

Chen, Y., Sasai, N., Ma, G., Yue, T., Jia, J., Briscoe, J., et al. (2011). Sonic Hedgehog Dependent Phosphorylation by CK1 $a$ and GRK2 Is Required for Ciliary Accumulation and Activation of Smoothened. Plos Biol. 9, e1001083. doi:10. 1371/journal.pbio.1001083

Corbit, K. C., Aanstad, P., Singla, V., Norman, A. R., Stainier, D. Y. R., and Reiter, J. F. (2005). Vertebrate Smoothened Functions at the Primary Cilium. Nature 437, 1018-1021. doi:10.1038/nature04117

Denef, N., Neubüser, D., Perez, L., and Cohen, S. M. (2000). Hedgehog Induces Opposite Changes in Turnover and Subcellular Localization of Patched and Smoothened. Cell 102, 521-531. doi:10.1016/s0092-8674(00)00056-8

Desai, P. B., Stuck, M. W., Lv, B., and Pazour, G. J. (2020). Ubiquitin Links Smoothened to Intraflagellar Transport to Regulate Hedgehog Signaling. J. Cell Biol. 219. doi:10.1083/jcb.201912104

di Magliano, M. P., and Hebrok, M. (2003). Hedgehog Signalling in Cancer Formation and Maintenance. Nat. Rev. Cancer 3, 903-911. doi:10.1038/ nrc1229

Fan, J., Jiang, K., Liu, Y., and Jia, J. (2013). Hrs Promotes Ubiquitination and Mediates Endosomal Trafficking of Smoothened in Drosophila Hedgehog Signaling. PLoS One 8, e79021. doi:10.1371/journal.pone.0079021

Fan, J., Liu, Y., and Jia, J. (2012). Hh-induced Smoothened Conformational Switch Is Mediated by Differential Phosphorylation at its C-Terminal Tail in a Doseand Position-dependent Manner. Developmental Biol. 366, 172-184. doi:10. 1016/j.ydbio.2012.04.007

Geiss-Friedlander, R., and Melchior, F. (2007). Concepts in Sumoylation: a Decade on. Nat. Rev. Mol. Cell Biol 8, 947-956. doi:10.1038/nrm2293

\section{AUTHOR CONTRIBUTIONS}

$\mathrm{JHJ}$ and JJ wrote the paper manuscript and prepare the figures.

\section{FUNDING}

This study was supported by the National Institutes of Health (R35GM131807 to JHJ; R35GM118063 to JJ), the Welch foundation grant (I-1603) to JJ and JHJ was also supported by the Shared Resource Facilities of the University of Kentucky Markey Cancer Center (P30CA177558), and the Imaging Core of the COBRE (P20GM121327).

Guha, M. (2012). Hedgehog Inhibitor Gets Landmark Skin Cancer Approval, but Questions Remain for Wider Potential. Nat. Rev. Drug Discov. 11, 257-258. doi: $10.1038 / \mathrm{nrd} 3714$

Happ, J. T., Arveseth, C. D., Bruystens, J., Bertinetti, D., Nelson, I. B., Olivieri, C., et al. (2021). A PKA Inhibitor Motif within Smoothened Controls Hedgehog Signal Transduction. bioRxiv. doi:10.1101/2021.07.05.451193

Hershko, A., and Ciechanover, A. (1998). The Ubiquitin System. Annu. Rev. Biochem. 67, 425-479. doi:10.1146/annurev.biochem.67.1.425

Ingham, P. W., and McMahon, A. P. (2001). Hedgehog Signaling in Animal Development: Paradigms and Principles. Genes Dev. 15, 3059-3087. doi:10. 1101/gad.938601

Ingham, P. W., Nakano, Y., and Seger, C. (2011). Mechanisms and Functions of Hedgehog Signalling across the Metazoa. Nat. Rev. Genet. 12, 393-406. doi:10. $1038 / \mathrm{nrg} 2984$

Irannejad, R., and von Zastrow, M. (2014). GPCR Signaling along the Endocytic Pathway. Curr. Opin. Cell Biol. 27, 109-116. doi:10.1016/j.ceb.2013.10.003

Jia, J., and Jiang, J. (2006). Decoding the Hedgehog Signal in Animal Development. Cell. Mol. Life Sci. 63, 1249-1265. doi:10.1007/s00018-005-5519-z

Jia, J., Tong, C., Wang, B., Luo, L., and Jiang, J. (2004). Hedgehog Signalling Activity of Smoothened Requires Phosphorylation by Protein Kinase A and Casein Kinase I. Nature 432, 1045-1050. doi:10.1038/nature03179

Jiang, J. (2021). Hedgehog Signaling Mechanism and Role in Cancer. Semin. Cancer Biol. doi:10.1016/j.semcancer.2021.04.003

Jiang, J., and Hui, C.-c. (2008). Hedgehog Signaling in Development and Cancer. Developmental Cell 15, 801-812. doi:10.1016/j.devcel.2008.11.010

Jiang, K., and Jia, J. (2015). Smoothened Regulation in Response to Hedgehog Stimulation. Front. Biol. 10, 475-486. doi:10.1007/s11515-015-1385-8

Jiang, K., Liu, Y., Fan, J., Epperly, G., Gao, T., Jiang, J., et al. (2014). Hedgehogregulated Atypical PKC Promotes Phosphorylation and Activation of Smoothened and Cubitus Interruptus inDrosophila. Proc. Natl. Acad. Sci. USA 111, E4842-E4850. doi:10.1073/pnas.1417147111

Jiang, K., Liu, Y., Zhang, J., and Jia, J. (2018). An Intracellular Activation of Smoothened Independent of Hedgehog Stimulation in Drosophila. J. Cell Sci 131. doi:10.1242/jcs.211367

Jiang, W., Yao, X., Shan, Z., Li, W., Gao, Y., and Zhang, Q. (2019). E3 Ligase Herc4 Regulates Hedgehog Signalling through Promoting Smoothened Degradation. J. Mol. Cell Biol 11, 791-803. doi:10.1093/jmcb/mjz024

Johnson, E. S. (2004). Protein Modification by SUMO. Annu. Rev. Biochem. 73, 355-382. doi:10.1146/annurev.biochem.73.011303.074118

Komander, D., and Rape, M. (2012). The Ubiquitin Code. Annu. Rev. Biochem. 81, 203-229. doi:10.1146/annurev-biochem-060310-170328

Kong, J. H., Young, C. B., Pusapati, G. V., Patel, C. B., Ho, S., Krishnan, A., et al. (2020). A Membrane-Tethered Ubiquitination Pathway Regulates Hedgehog Signaling and Heart Development. Developmental Cell 55, 432-449. doi:10. 1016/j.devcel.2020.08.012

Li, S., Chen, Y., Shi, Q., Yue, T., Wang, B., and Jiang, J. (2012). Hedgehog-regulated Ubiquitination Controls Smoothened Trafficking and Cell Surface Expression in Drosophila. Plos Biol. 10, e1001239. doi:10.1371/journal.pbio.1001239

Li, S., Cho, Y. s., Wang, B., Li, S., and Jiang, J. (2018a). Regulation of Smoothened Ubiquitination and Cell Surface Expression by a Cul4-DDB1-G $\beta$ E3 Ubiquitin Ligase Complex. J. Cell Sci 131. doi:10.1242/jcs.218016 
Li, S., Li, S., Han, Y., Tong, C., Wang, B., Chen, Y., et al. (2016). Regulation of Smoothened Phosphorylation and High-Level Hedgehog Signaling Activity by a Plasma Membrane Associated Kinase. Plos Biol. 14, e1002481. doi:10.1371/ journal.pbio. 1002481

Li, S., Li, S., Wang, B., and Jiang, J. (2018b). Hedgehog Reciprocally Controls Trafficking of Smo and Ptc through the Smurf Family of E3 Ubiquitin Ligases. Sci. Signal. 11. doi:10.1126/scisignal.aan 8660

Li, S., Ma, G., Wang, B., and Jiang, J. (2014). Hedgehog Induces Formation of PKASmoothened Complexes to Promote Smoothened Phosphorylation and Pathway Activation. Sci. Signal. 7, ra62. doi:10.1126/scisignal.2005414

Lv, B., Stuck, M. W., Desai, P. B., Cabrera, O. A., and Pazour, G. J. (2021). E3 Ubiquitin Ligase Wwp1 Regulates Ciliary Dynamics of the Hedgehog Receptor Smoothened. J. Cell Biol. 220. doi:10.1083/jcb.202010177

Ma, G., Li, S., Han, Y., Li, S., Yue, T., Wang, B., et al. (2016). Regulation of Smoothened Trafficking and Hedgehog Signaling by the SUMO Pathway. Developmental Cell 39, 438-451. doi:10.1016/j.devcel.2016.09.014

Matusek, T., Wendler, F., Polès, S., Pizette, S., D’Angelo, G., Fürthauer, M., et al. (2014). The ESCRT Machinery Regulates the Secretion and Long-Range Activity of Hedgehog. Nature 516, 99-103. doi:10.1038/nature13847

Pietrobono, S., and Stecca, B. (2018). Targeting the Oncoprotein Smoothened by Small Molecules: Focus on Novel Acylguanidine Derivatives as Potent Smoothened Inhibitors. Cells 7, 272. doi:10.3390/cells7120272

Popovic, D., Vucic, D., and Dikic, I. (2014). Ubiquitination in Disease Pathogenesis and Treatment. Nat. Med. 20, 1242-1253. doi:10.1038/nm.3739

Ranieri, N., Thérond, P. P., and Ruel, L. (2014). Switch of PKA Substrates from Cubitus Interruptus to Smoothened in the Hedgehog Signalosome Complex. Nat. Commun. 5, 5034. doi:10.1038/ncomms6034

Reyes-Turcu, F. E., Ventii, K. H., and Wilkinson, K. D. (2009). Regulation and Cellular Roles of Ubiquitin-specific Deubiquitinating Enzymes. Annu. Rev. Biochem. 78, 363-397. doi:10.1146/annurev.biochem.78.082307.091526

Rohatgi, R., Milenkovic, L., and Scott, M. P. (2007). Patched1 Regulates Hedgehog Signaling at the Primary Cilium. Science 317, 372-376. doi:10.1126/science. 1139740

Sekulic, A., and Von Hoff, D. (2016). Hedgehog Pathway Inhibition. Cell 164, 831. doi:10.1016/j.cell.2016.02.021

Shenoy, S. K., McDonald, P. H., Kohout, T. A., and Lefkowitz, R. J. (2001). Regulation of Receptor Fate by Ubiquitination of Activated $\beta 2$-Adrenergic Receptor and $\beta$-Arrestin. Science 294, 1307-1313. doi:10.1126/science. 10638661063866

Shinde, S. R., Nager, A. R., and Nachury, M. V. (2020). Ubiquitin Chains Earmark GPCRs for BBSome-Mediated Removal from Cilia. J. Cell Biol. 219. doi:10. 1083/jcb.202003020

Sun, X., Sun, B., Cui, M., and Zhou, Z. (2019). HERC4 Exerts an Anti-tumor Role through Destabilizing the Oncoprotein Smo. Biochem. Biophysical Res. Commun. 513, 1013-1018. doi:10.1016/j.bbrc.2019.04.113

Taipale, J., and Beachy, P. A. (2001). The Hedgehog and Wnt Signalling Pathways in Cancer. Nature 411, 349-354. doi:10.1038/35077219

Villavicencio, E. H., Walterhouse, D. O., and Iannaccone, P. M. (2000). The Sonic Hedgehog-Patched-Gli Pathway in Human Development and Disease. Am. J. Hum. Genet. 67, 1047-1054. doi:10.1016/s0002-9297(07)62934-6
Wojcikiewicz, R. J. H. (2004). Regulated Ubiquitination of Proteins in GPCRInitiated Signaling Pathways. Trends Pharmacol. Sci. 25, 35-41. doi:10.1016/j. tips.2003.11.008

Xia, R., Jia, H., Fan, J., Liu, Y., and Jia, J. (2012). USP8 Promotes Smoothened Signaling by Preventing its Ubiquitination and Changing its Subcellular Localization. Plos Biol. 10, e1001238. doi:10.1371/journal.pbio.1001238

Xie, J., Murone, M., Luoh, S.-M., Ryan, A., Gu, Q., Zhang, C., et al. (1998). Activating Smoothened Mutations in Sporadic Basal-Cell Carcinoma. Nature 391, 90-92. doi:10.1038/34201

Yang, L., Xie, G., Fan, Q., and Xie, J. (2010). Activation of the Hedgehog-Signaling Pathway in Human Cancer and the Clinical Implications. Oncogene 29, 469-481. doi:10.1038/onc.2009.392

Yang, X., Mao, F., Lv, X., Zhang, Z., Fu, L., Lu, Y., et al. (2013). Drosophila Vps36 Is Involved in Hh Signaling by Regulating Smo Trafficking. J. Cell Sci 126, 4230-4238. doi:10.1242/jcs.128603

Yue, S., Tang, L.-Y., Tang, Y., Tang, Y., Shen, Q.-H., Ding, J., et al. (2014). Requirement of Smurf-Mediated Endocytosis of Patched1 in Sonic Hedgehog Signal Reception. eLife 3. doi:10.7554/eLife.02555

Zhang, J., Liu, Y., Jiang, K., and Jia, J. (2017). SUMO Regulates the Activity of Smoothened and Costal-2 in Drosophila Hedgehog Signaling. Sci. Rep. 7, 42749. doi:10.1038/srep42749

Zhang, Q., and Jiang, J. (2021). Regulation of Hedgehog Signal Transduction by Ubiquitination and Deubiquitination. Ijms 22 (24), 13338. doi:10.3390/ ijms222413338

Zhao, Y., Tong, C., and Jiang, J. (2007). Transducing the Hedgehog Signal across the Plasma Membrane. Fly 1, 333-336. doi:10.4161/fly.5570

Zheng, X., Mann, R. K., Sever, N., and Beachy, P. A. (2010). Genetic and Biochemical Definition of the Hedgehog Receptor. Genes Dev. 24, 57-71. doi:10.1101/gad.1870310

Zhou, Z., Yao, X., Pang, S., Chen, P., Jiang, W., Shan, Z., et al. (2018). The Deubiquitinase UCHL5/UCH37 Positively Regulates Hedgehog Signaling by Deubiquitinating Smoothened. J. Mol. Cell Biol 10, 243-257. doi:10.1093/jmcb/ mjx036

Conflict of Interest: The authors declare that the research was conducted in the absence of any commercial or financial relationships that could be construed as a potential conflict of interest.

Publisher's Note: All claims expressed in this article are solely those of the authors and do not necessarily represent those of their affiliated organizations, or those of the publisher, the editors and the reviewers. Any product that may be evaluated in this article, or claim that may be made by its manufacturer, is not guaranteed or endorsed by the publisher.

Copyright (C) 2022 Jia and Jiang. This is an open-access article distributed under the terms of the Creative Commons Attribution License (CC BY). The use, distribution or reproduction in other forums is permitted, provided the original author $(s)$ and the copyright owner(s) are credited and that the original publication in this journal is cited, in accordance with accepted academic practice. No use, distribution or reproduction is permitted which does not comply with these terms. 